\title{
MicroRNA-302b Suppresses Human Epithelial Ovarian Cancer Cell Growth by Targeting RUNX1
}

Tingting Ge Mingzhu Yin Meng Yang Tianbo Liu Ge Lou

Department of Gynecology, the Affiliated Tumor Hospital, Harbin Medical University, Harbin China

\section{Key Words}

miR-302b • RUNX1 • Epithelial ovarian carcinoma $\bullet$ STAT3

\begin{abstract}
Background: The microRNA (miR)-302 family functions as a tumor suppressor in human cancer. However, its role in epithelial ovarian carcinoma (EOC) remains unknown. Here, we investigated the role of miR-302b and its target gene RUNX1 in EOC. Methods: The expression levels of miR-302b and RUNX1 were assessed by quantitative real-time PCR and western blotting. The effects of ectopic expression of miR-302b were evaluated by the MTT assay, colony forming assay and flow cytometry. RUNX1 was identified as a target of miR-302b and their interaction was confirmed by luciferase activity assays, RUNX1 silencing and overexpression of a RUNX1 mutant construct lacking the 3'UTR. The effect of miR-302b on the suppression of tumor growth was investigated in vivo in a xenograft mouse model. Results: MiR-302b levels were markedly decreased in EOC specimens. Ectopic expression of miR-302b in EOC cells inhibited cell proliferation and colony formation, induced G0/G1 arrest, and promoted apoptosis. RUNX1 was identified as a direct target of miR-302b, and knockdown of RUNX1 inhibited cell growth in a manner similar to miR-302b overexpression, whereas introduction of a 3'UTR mutant of RUNX1 reversed the suppressive effect of miR-302b. Furthermore, miR$302 \mathrm{~b}$ overexpression led to the inactivation of the STAT3 signaling pathway in EOC cells and inhibited tumor growth in a xenograft mouse model. Conclusions: MiR-302b functions as a tumor suppressor in EOC by targeting RUNX1 and modulating the activity of the STAT3 signaling pathway.
\end{abstract}




\section{Introduction}

Runt-related transcription factor 1 (RUNX1) belongs to a small family of heterodimeric transcription factors involved in cell lineage determination that play a role in various types of cancer by activating or repressing the transcription of key regulators of growth, survival and differentiation [1]. RUNX1 is one of the most frequently mutated genes in human leukemias [2]. RUNX proteins have been implicated in the transcriptional regulation of cell cycle related proteins such as Cyclin D1 and p21, and RUNX expression levels vary during the cell cycle. RUNX1 levels increase by two- to four-fold during the $S$ and G2/M phases compared to the G1 phase in hematopoietic cells, and experiments have suggested that RUNX1 is a positive regulator of the G1 to $S$ transition $[3,4]$. RUNX1 can act as a tumor suppressor or an oncogene in different cells and tissues through the regulation of cancerrelated genes such as p21 and signal transducer and activator of transcription 3 (STAT3) [5]. RUNX1 represses p21 and promotes the activation of STAT3, a transcription factor with oncogenic properties, by facilitating its phosphorylation through the Jak/Stat pathway [6, 7]. Phosphorylation of STAT3 on its conserved tyrosine residue promotes its homodimerization and translocation to the nucleus to regulate the transcription of genes involved in survival, proliferation and invasion, and constitutive activation of STAT3 is associated with malignant transformation and poor clinical outcomes in several cancers [8-10]. In keratinocytes and skin cancer cells, RUNX1 maintains STAT3 in an active state by repressing cytokine signaling suppressors (SOCS) [6]. Recently, RUNX1 was shown to be upregulated in human epithelial ovarian carcinoma (EOC) tissues and associated with EOC cell proliferation, migration and invasion [11].

MicroRNAs (miRNAs) are small (22-nucleotide) noncoding single stranded RNAs that regulate gene expression by binding to the $3^{\prime}$ untranslated region ( $3^{\prime}$ UTR) of their target mRNAs, modulating mRNA stability and/or translation [12]. An increasing body of evidence suggests that miRNAs play a role in the pathogenesis of cancer through the modulation of genes and signaling pathways involved in tumorigenesis [13]. In EOC, miRNA profiling studies have identified miRNAs associated with tumor progression and chemotherapy resistance [14-16]. MiR-302b is a member of the miR-302 cluster, which consists of four homologous miRNAs that are transcribed as a noncoding RNA cluster and are specifically expressed in human and mouse embryonic stem cells [17]. MiR-302b acts as a tumor suppressor in esophageal squamous cell carcinoma and it is downregulated in hepatocellular carcinoma, where it inhibits cell proliferation by targeting EGFR $[18,19]$. Although genome-wide miRNA expression profiling studies showed that miR-302b is downregulated in ovarian carcinoma $[14,20]$, the exact role of miR-302b in EOC remains unknown.

In the present study, we showed that miR-302b is downregulated in EOC tissues compared to normal ovarian tissues. Ectopic expression of miR-302b inhibited cell proliferation and colony formation, induced G0/G1 cell cycle arrest and apoptosis in ovarian cancer cells, and inhibited tumor formation in a xenograft mouse model. We identified RUNX1 as a direct target of miR-302b, and showed that its tumor suppressor effect is mediated by the downregulation of RUNX1 and the inhibition of STAT3 signaling.

\section{Materials and Methods}

Clinical specimens and cell lines

This study was approved by the Ethics Committee of Harbin Medical University. Animal experiments were performed with the approval of the Animal Ethical Committee of Harbin Medical University and in conformity with national guidelines for the care and use of laboratory animals. Human EOC specimens and normal ovarian tissues were collected from Affiliated Tumor Hospital of Harbin Medical University after obtaining written informed consent from all patients. Tissues were snap-frozen in liquid nitrogen and stored at $-80^{\circ} \mathrm{C}$ following surgery for qRT-PCR. The human EOC cell lines SKOV3 and OVCAR3 were purchased from 
American Type Culture Collection (ATCC; Manassas, VA, USA) and were maintained in McCoy's 5A medium and RPMI-1640, respectively, supplemented with $10 \%$ fetal bovine serum (FBS).

\section{Quantitative real-time RT-PCR ( $q R T-P C R$ )}

Total RNA was extracted using the TRIzol reagent (Life Technologies, Inc.) according to the manufacturer's instructions. For miR-302b analysis, RT primer and real-time primer sequences used were as follows: RT primer, 5'-GTCGTATCCAGTCCAGGGACCGAGGACTGGATACGACCTACTA-3'; miR-302b forward, 5'-GCGTAAGTGCTTCCATGTT-3' and miR-302b reverse, 5'-TCCAGGGACCGAGGA-3'. For the detection of RUNX1 expression, the following primers were used: RUNX1 forward, $5^{\prime}$-CGAAGACATCGGCAGAAACT-3' and reverse, $5^{\prime}$-TAAAGGCAGTGGAGTGGTTCA- $3^{\prime}$. U6 and $\beta$-actin were used to normalize miRNA and mRNA respectively. All RT-PCR experiments were performed on a BioRad iQ5 Real-Time PCR Detection System (Bio-Rad, Hercules, CA, USA). The expression levels relative to U6 and $\beta$-actin were calculated using the formula $2^{-\Delta \Delta C T}$.

\section{Western blotting}

For western blot analysis, protein lysates were prepared by resuspending cell pellets in Laemmli sample buffer containing 5\% $\beta$-mercaptoethanol. Lysates were separated in 6-12\% Tris-glycine gel electrophoresis and transferred to polyvinylidene difluoride membranes. After blocking in 5\% nonfat dry milk in TBST ( $20 \mathrm{mmol} / \mathrm{L}$ TRIS- $\mathrm{HCl}, 0.5 \mathrm{M} \mathrm{NaCl}$ and $0.1 \%$ Tween 20), membranes were incubated in primary antibodies at $4^{\circ} \mathrm{C}$ overnight, followed by three $15 \mathrm{~min}$ washes in TBST and incubation in horseradish peroxidase-conjugated secondary antibodies and signal detection using the enhanced chemiluminescence system (Thermo Fisher Scientific). Primary antibodies used were as follows: anti-RUNX1 (1:200), anti-Bcl-2 (1:500), anti-Bax (1:200), anti- $\beta$-actin (1:5,000) (Santa Cruz Biotechnology, Santa Cruz, CA, USA); antiphospho-STAT3 (Tyr705) (1:1000), anti-Cyclin D1 (1:1000), and anti-Cleaved caspase-3 (Asp175) (1:1000) (Cell Signaling Technology, Danvers, MA, USA).

\section{Generation of stable miR-302b-experssing EOC cells}

A miR-302b-expression vector was generated by PCR-amplifying a DNA fragment encoding the miR-302b-precursor using the following primers: forward, 5'-CGGGATCCGCTCCCTTCAACTTTAACATGGAAGTGCTTTCTGTGACTTTAAA-3' and reverse, 5'-CCCAAGCTTACTCCTACTAAAACATGGAAGCACTTACTTTTAAAGTC-3', and cloning it into the BamHI and HindIII sites of the pSilencer vector (Life Technologies). The miR-302b carrying and control vectors were transfected into SKOV3 or OVCAR3 cells using Lipofectamine 2000 (Invitrogen, Carlsbad, CA, USA), followed by selection with neomycin (Gibco, Grand Island, NY, USA) to generate stable cell lines.

Vector construction and luciferase reporter assay

The $3^{\prime}$ UTR region of RUNX1 containing the wild-type or mutant potential target site for miR-302b was synthesized and inserted into the pMIR-reporter vector (Ambion, Carlsbad, CA, USA) at Spe I and Hind III sites. The RUNX1 3'UTR oligos were synthesized as follows: RUNX1wt $3^{\prime}$ UTR, sense, 5'-CTAGTATTATTTGTTTTACACACATGCAGTAGCACTTTGGTAAGAA- ${ }^{\prime}$ and antisense, $\quad 5^{\prime}$-AGCTTTCTTACCAAAGTGCTACTGCATGTGTGTAAAACAAATAATA-3'; $\quad$ RUNX1-mut 3'UTR, sense, 5'-CTAGTATTATTTGTTTTACACAGTAGCAGTACGTGATTGGTAAGAA-3' and antisense, 5'-AGCTTTCTTACCAATCACGTACTGCTACTGTGTAAAACAAATAATA-3'. For luciferase assay, SKOV3 cells were co-transfected with RUNX1-wt 3'UTR or RUNX1-mut 3'UTR and miR-302b or anti-miR-302b (Ambion) or control using Lipofectamine 2000 (Invitrogen). Cell lysates were prepared $24 \mathrm{~h}$ after transfection, and luciferase activity was measured using a Dual-Glo Luciferase Assay System (Promega, Madison, WI, USA).

RUNX1-expression vector without miR-302b binding sites

A full-length RUNX1 cDNA lacking the 3'UTR was generated by PCR amplification using the following primers: sense, 5'-GCGAATTCGCCACCATGCGTATCCCCGTAGA-3' and antisense, 5'-GCAGCCTCGAGGCTCAGTAGGGCCTCCACA-3'. The construct was inserted into the pcDNA3.1(+) (Invitrogen) vector between the EcoR I and Xho I sites to generate a RUNX1 expression vector without miR$302 \mathrm{~b}$ binding sites. RUNX1 siRNA and negative control oligonucleotides were purchased from GenePharma (Shanghai, China). 
Cell Proliferation Assay

SKOV3 or OVCAR3 cells $\left(2 \times 10^{4}\right.$ cells/ml) were seeded in 96-well plates $24 \mathrm{~h}$ post-transfection. 3-(4,5-dimethylthiazol-2-yl)-2,5-diphenyl-tetrazolium bromide (MTT, $20 \mu \mathrm{l}, 5 \mathrm{mg} / \mathrm{ml}$ ) (Sigma-Aldrich, St Louis, MO, USA) was added to each well and cells were incubated for another $4 \mathrm{~h}$. The reaction was stopped by addition of $150 \mu \mathrm{l}$ DMSO. The absorbance of the solution was read at $570 \mathrm{~nm}$ using an lQuant Universal Microplate Spectrophotometer (BioTek, Winooski, VT, USA).

\section{Colony formation}

SKOV3 or OVCAR3 cells were treated as indicated and seeded at a density of 200 cells per $35-\mathrm{mm}$ culture dish. After 14 days, the dishes were washed twice with PBS, fixed with cold methanol, stained with Giemsa solution (Sigma-Aldrich) for $15 \mathrm{~min}$, washed with water and air-dried. The number of colonies was determined by imaging with a Multimage ${ }^{\mathrm{TM}}$ Cabinet (Alpha Innotech Corporation).

\section{Cell cycle analysis}

SKOV3 or OVCAR3 cells were harvested $48 \mathrm{~h}$ post-transfection, washed with prechilled PBS, fixed with $70 \%$ ethanol and stored at $4{ }^{\circ} \mathrm{C}$ overnight. Cells were rehydrated with PBS for $10 \mathrm{~min}$ and stained with a propidium iodide (PI) solution containing $50 \mu \mathrm{g} / \mathrm{ml} \mathrm{PI,} 2 \mu \mathrm{g} / \mathrm{ml}$ DNase-free RNase A and $0.2 \%$ NP-40 in PBS for $15 \mathrm{~min}$ at $37^{\circ} \mathrm{C}$. Cell cycle analysis was performed using a FACSCalibur flow cytometer (BD Biosciences, San Jose, CA, USA).

\section{Apoptosis assay}

For detection of apoptotic cells, SKOV3 or OVCAR3 cells were harvested $48 \mathrm{~h}$ post-transfection, washed twice with prechilled PBS and resuspended in $100 \mu \mathrm{L}$ binding buffer Annexin V and PI double staining was performed using the Annexin V-FITC Apoptosis Detection kit (BD Biosciences) and flow cytometric analysis was performed on a FACSCalibur flow cytometer (BD Biosciences).

\section{Tumor xenograft model and tumorigenicity assay}

SKOV3 cells $\left(5 \times 10^{6}\right.$ cells $\left./ \mathrm{ml}\right)$ stably transfected with miR-302b or control vector were subcutaneously injected into 4-week-old male nude mice. Tumor volume and weight was monitored over a period of 32 days. Tumor samples were analyzed by immunohistochemistry using anti-Ki-67 antibody (1:100) and antiPCNA antibody (1:100, Dako, Carpinteria, CA, USA). MiR-302b and RUNX1 levels in xenograft tumor tissues were analyzed by qRT-PCR and western blot, respectively.

\section{Statistical analysis}

All results were expressed as mean \pm SD. Data analysis was performed using SPSS 17.0 (SPSS Inc., Chicago, IL, USA). Statistical significance was analyzed using Student's t test or one-way ANOVA. Differences with $P<0.05$ were considered statistically significant.

\section{Results}

Decreased miR-302b and increased RUNX1 expression in human EOC specimens

The expression of miR-302b and RUNX1 was examined in 38 EOC and 16 normal ovarian tissues by qRT-PCR. The results showed significantly lower levels of miR-302b and significantly higher levels of RUNX1 mRNA in EOC tissues than in normal ovarian tissues $(P<0.001$ both $)$ (Fig. 1A and B). Western blot analysis of RUNX1 protein expression showed a significant upregulation of RUNX1 in EOC compared to normal ovarian tissues $(P<0.01)$. A representative blot and quantitative analysis of protein levels by densitometry are shown in Fig. 1C.

MiR-302b inhibits growth and promotes apoptosis in EOC cells

To determine the effect of miR-302b on EOC cell viability, SKOV3 or OVCAR3 ovarian cancer cells were transfected with miR-302b expressing vector, anti-miR-302b or their respective controls. The miR- $302 \mathrm{~b}$ vector caused a more than 20 -fold upregulation of the 
Fig. 1. Dysregulation of miR302b/RUNX1 expression in EOC tissues. A. Quantitative analysis of miR-302b expression in EOC tissues $(\mathrm{n}=38)$ and normal ovarian tissues $(\mathrm{n}=16)$ by qRTPCR normalized to U6 snRNA. B. Quantitative analysis of RUNX1 expression in EOC tissues ( $\mathrm{n}=$ 38) and normal ovarian tissues $(n=16)$ by qRT-PCR normalized to $\beta$-actin. C. Western blot analysis of RUNX1 expression in normal ovarian tissues and EOC tissues (representative blots are shown). ${ }^{* *} P<0.01,{ }^{* * *} P<0.001$.

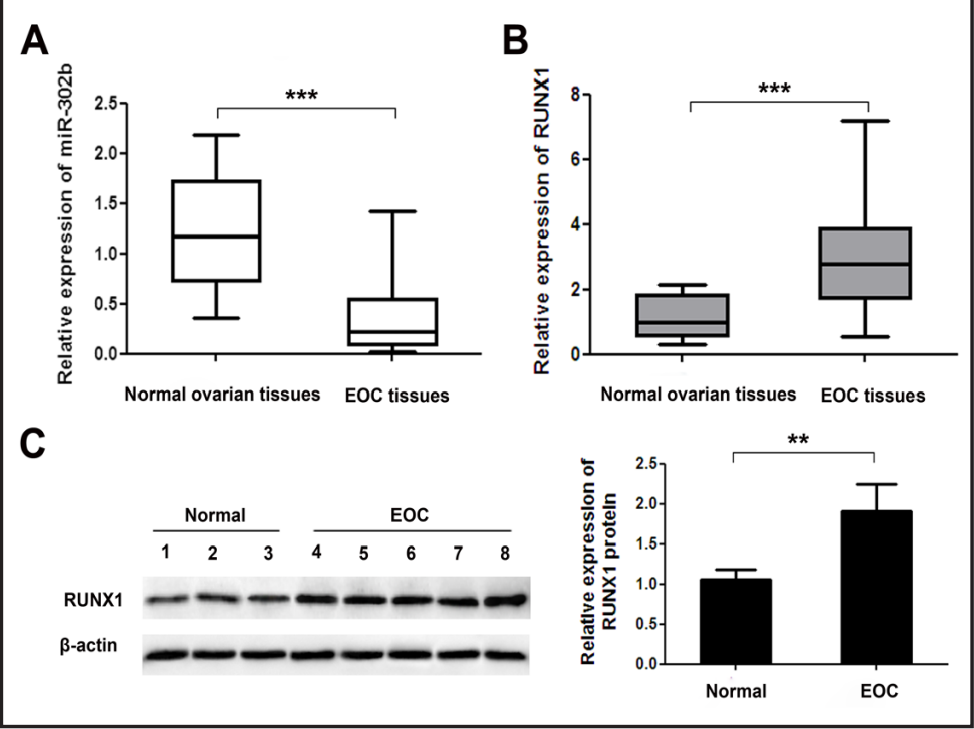

expression of miR-302b compared to the miR-control in both cell lines, whereas anti-miR$302 \mathrm{~b}$ significantly downregulated the levels of miR-302b $(P<0.001)$ (Fig. 2A). Assessment of cell viability over a period of $72 \mathrm{~h}$ using the MTT assay showed significant inhibition of cell growth in miR-302b transfected cells in both cell lines $(P<0.01)$, whereas the anti-miR$302 \mathrm{~b}$ construct significantly stimulated cell growth compared to the respective controls $(P<0.01)$ (Fig. 2B). Similar results were obtained in the colony formation assay, in which ectopic expression of miR-302b significantly inhibited colony forming ability compared to the controls in SKOV3 and OVCAR3 cells, whereas anti-miR-302b had the opposite effect $(P<0.01)$ (Fig. 2C). Analysis of cell cycle distribution showed that miR-302b significantly increased the number of cells at G0/G1 phase, with a concomitant decrease in the proportion of cells in S phase, and anti-miR-302b had the opposite effect (Fig. 2D). To determine the mechanism of cell death induced by miR-302b, SKOV3 or OVCAR3 cells transfected as described above were analyzed by Annexin V-FITC/PI flow cytometry. The results indicated that overexpression of miR-302b significantly increased the rate of apoptosis, whereas downregulation of its expression by anti-miR-302b had the opposite effect (Fig. 2E). Taken together, these results indicated that miR-302b inhibits the growth and colony forming ability of EOC cells by inducing cell cycle arrest at G0/G1 phase and promoting apoptosis.

\section{MiR-302b directly targets RUNX1}

Conserved binding sites for miR-302b in the 3'UTR of RUNX1 were identified in several species by TargetScan, PicTar, and DIANA-microT and are shown in Fig. 3A. A mutant construct was generated by modifying eight residues in the 3'UTR of RUNX1. To verify RUNX1 as a direct target of miR-302b, constructs containing wild-type (wt) and mutant (mut) RUNX1 3'UTR were cloned into a luciferase reporter vector and co-transfected with miR-302b or anti-miR-302b and their respective negative controls into SKOV3 cells. Luciferase activity was significantly decreased by miR-302b mimics only in the presence of RUNX1-wt $3^{\prime}$ UTR, but not in cells carrying RUNX1-mut 3'UTR, and this effect was reversed by anti-miR-302b, confirming that miR-302b directly targets the 3'UTR of RUNX1 (Fig. 3B). To determine whether miR-302b affects the expression of RUNX1, miR-302b, anti-miR-302b and their respective negative controls were transfected into SKOV3 cells and the mRNA expression of RUNX1 was assessed by qRT-PCR, which showed a significant downregulation of RUNX1 expression by miR-302b, whereas anti-miR-302b had the opposite effect (Fig. 3C). Assessment of protein levels by western blotting showed that miR-302b downregulated RUNX1 protein levels by approximately two-fold, whereas anti-miR-302b caused an approximately two-fold increase in RUNX1 protein levels compared to the negative control $(P<0.01)$ (Fig. 3D). 


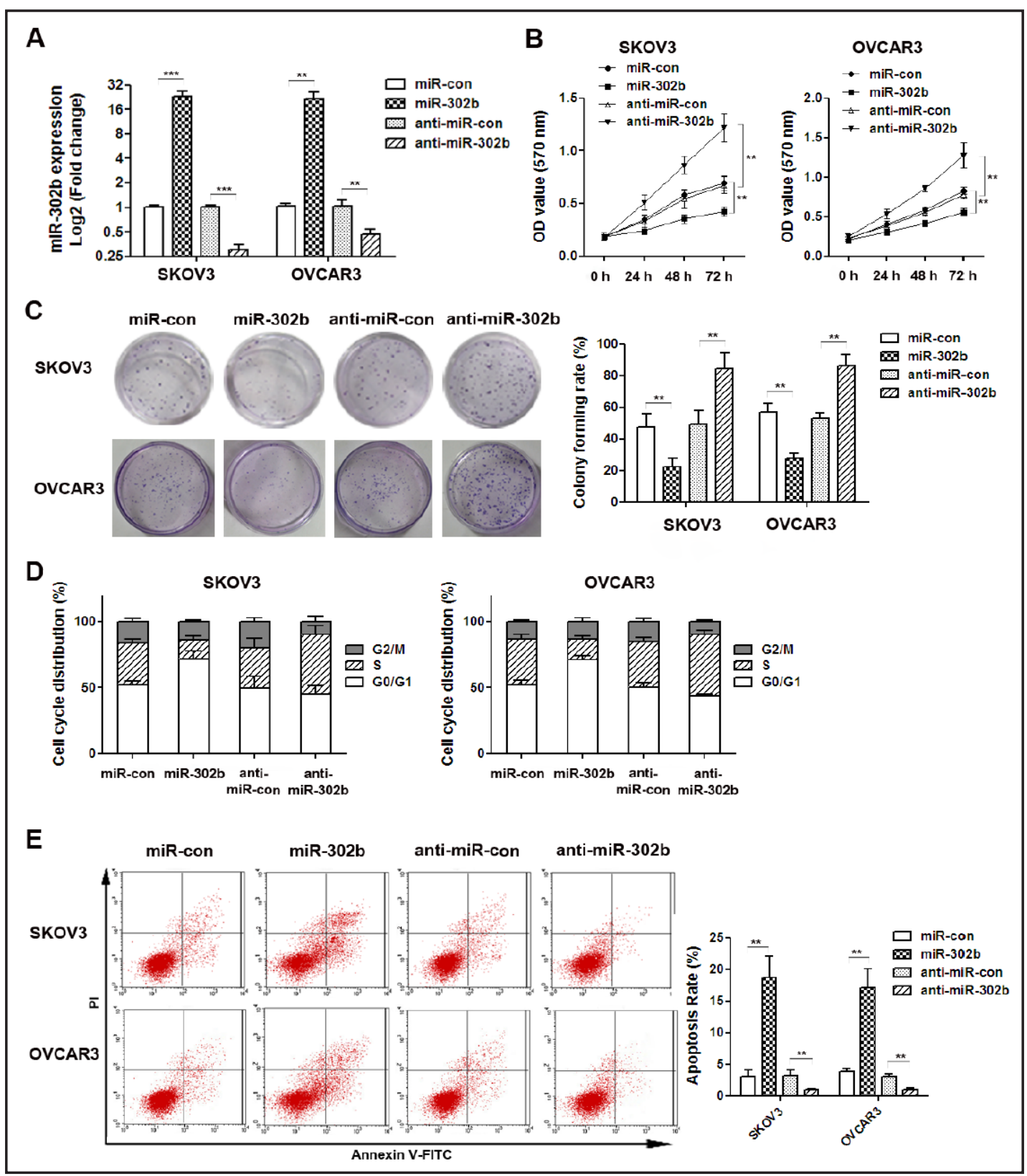

Fig. 2. Inhibition of cell growth and induction of apoptosis by miR-302b in ovarian cancer cells. A. MiR302b mRNA expression was determined by qRT-PCR in SKOV3 or OVCAR3 cells transfected with miR-302b, anti-miR-302b, or their respective controls. U6 snRNA was used for endogenous normalization. B. Effect of miR-302b on EOC cell viability was measured using the MTT assay. C. Colony formation assays. D. Cell-cycle distribution of SKOV3 or OVCAR3 cells transfected with miR-302b, anti-miR-302b, or their nonspecific controls for $48 \mathrm{~h}$. E. Flow cytometry and Annexin V assays show the number of apoptotic cells in the SKOV3 and OVCAR3 cell lines. Results represent the mean \pm SD. ${ }^{* *} P<0.01,{ }^{* * *} P<0.001$.

\section{RUNX1 mediates miR-302b induced growth inhibition and apoptosis promotion in SKOV3 cells}

To further examine the regulation of RUNX1 expression by miR-302b and its effect on cell growth and apoptosis, a RUNX1 expression vector lacking the miR-302b binding site was generated and transfected into SKOV3 cells ectopically expressing miR-302b. In addition, RUNX1 expression was knocked down using a specific siRNA. Western blot analysis 
A

Hsa 5'...uUacacacaugcaguagcacuUUgguaA...3'

Ptr 5'...UUACACACAUGCAGUAGCACUUUGgUAA....3'

Mml 5 '...UUACACACAUgCAgUagCaCUUUgGuaA....3'

Mmu 5'...UUACAUA - GAGCAGCAGCACUUUGGUAA....3'

Rno 5 '...UUACAUaAgagcagcagcacuUUgguaA....3'

Cpo 5 '...uUacauagaugcaguagcacuuUgguaA....3'

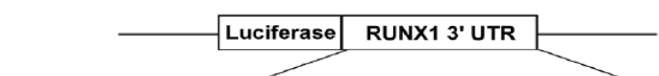

RUNX1-wt 3'UTR: 5'...UUACACACAUGCAGUAGCACUUU....3' |||| $\mid$ | || || | miR-302b 3'-GAUGAUUUUGUACCU - - UCGUGAAU-5' RUNX1-mut 3'UTR: 5'...UUACACAGUAGCAGUACGUCAUU...3'

B

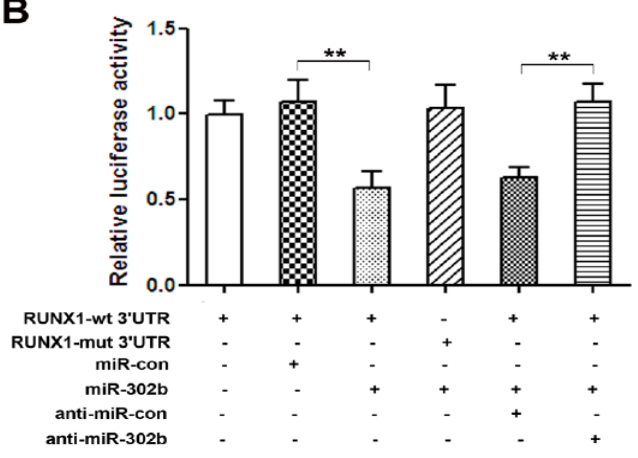

C
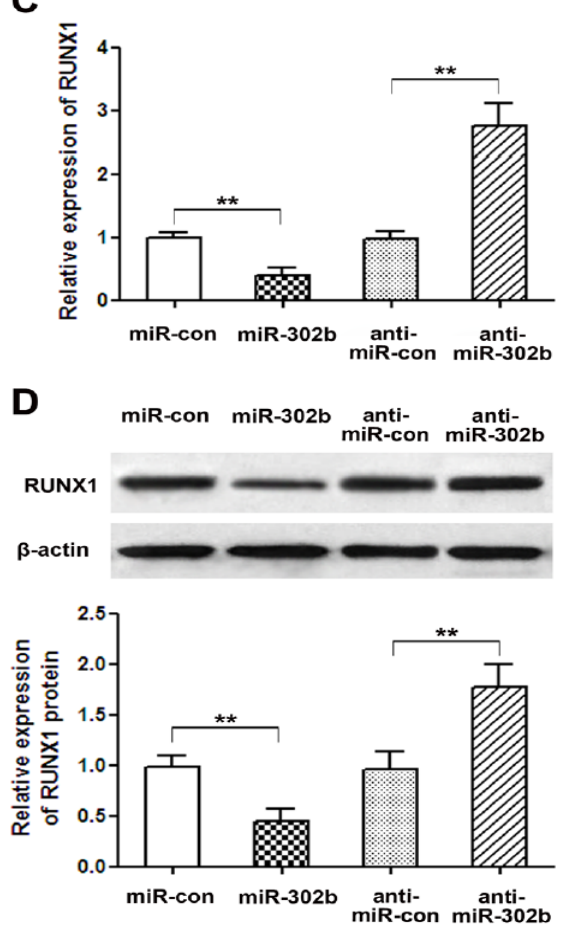

Fig. 3. RUNX1 is a direct target of miR-302b. A. Predicted miR-302b target sequence in the $3^{\prime} U T R$ of RUNX1 (RUNX1-wt 3'UTR) and a construct containing eight mutated nucleotides in the RUNX1 3'UTR (RUNX1-mut $3^{\prime}$ UTR). B. Luciferase reporter assay in SKOV3 cells transfected with reporter vectors containing wild-type (wt) or mutant (mut) RUNX1 3'UTR. Relative luciferase expression was normalized to Renilla activity. C and D, SKOV3 cells were transfected with miR-302b, anti-miR-302b, or their respective nonspecific controls and RUNX1 expression was assessed by qRT-PCR (C) and western blotting (D) and normalized to the expression of $\beta$-actin. Results represent mean $\pm \mathrm{SD}$. ${ }^{* *} P<0.01$.

showed that co-expression of miR-302b and empty vector significantly downregulated the expression of RUNX1 protein, whereas in the presence of the mutant RUNX1 construct, the levels of RUNX1 protein increased to a level above that of the control $(P<0.01)$ (Fig. 4A). SiRNA-mediated knockdown of RUNX1 significantly downregulated the expression of the RUNX1 protein, similar to the effect of miR-302b overexpression $(P<0.01)$ (Fig. 4A). We then examined the effects of these constructs on cell viability, colony forming ability, cell cycle progression and apoptosis induction. The results showed that while miR-302b overexpressing SKOV3 cells showed decreased cell viability, cell cycle arrest at the G0/G1 phase, decreased colony forming ability and a significantly increased rate of apoptosis, cotransfection with the mutant RUNX1 expression vector lacking the miR-302b 3'UTR binding site abolished these effects $(P<0.01$ all) (Fig. 4B-E). SiRNA mediated silencing of RUNX1 expression decreased cell viability, colony forming ability, caused cell cycle arrest at G0/G1 and increased the rate of apoptosis, similar to the effects of miR-302b overexpression (Fig. $4 \mathrm{~B}-\mathrm{E}$ ). Taken together, these results confirm that RUNX1 is a direct target of miR-302b and mediates its effects on cell growth and apoptosis in ovarian cancer.

\section{MiR-302b inhibits the STAT3 pathway}

STAT3 is frequently activated in ovarian cancer and promotes tumor growth and survival by upregulating the expression of oncogenes such as Cyclin D1 and antiapoptotic proteins such as Bcl-2. In addition, STAT3 activation is induced by the suppression of the expression of SOCS3 and 4 by RUNX1, supporting the role of RUNX1/STAT3 signaling in promoting cell proliferation and tumorigenesis [6]. Therefore, we examined the potential effect of miR-302b 
A
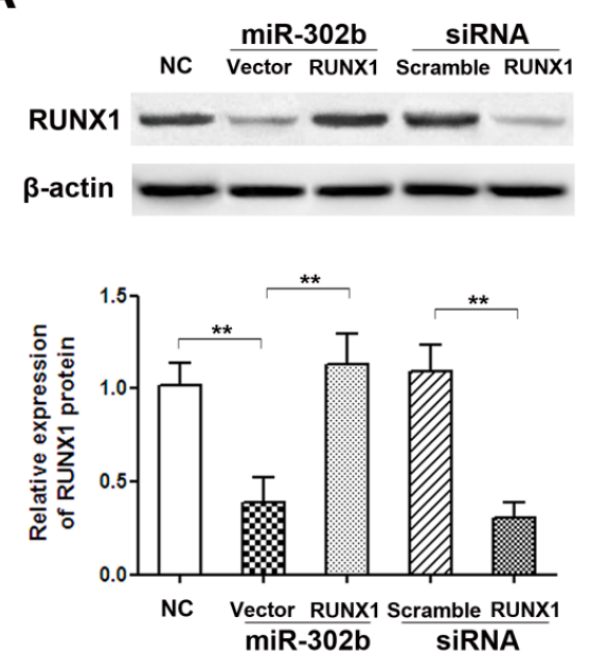

B

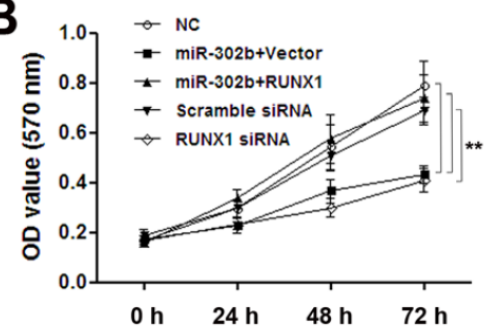

$D_{8}$

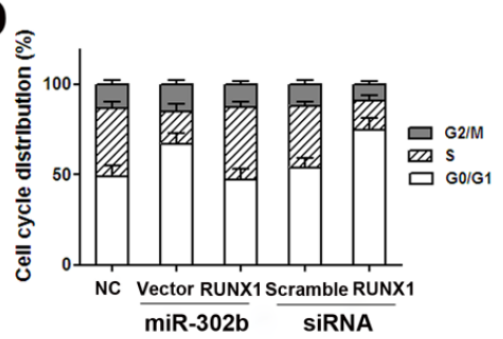

C
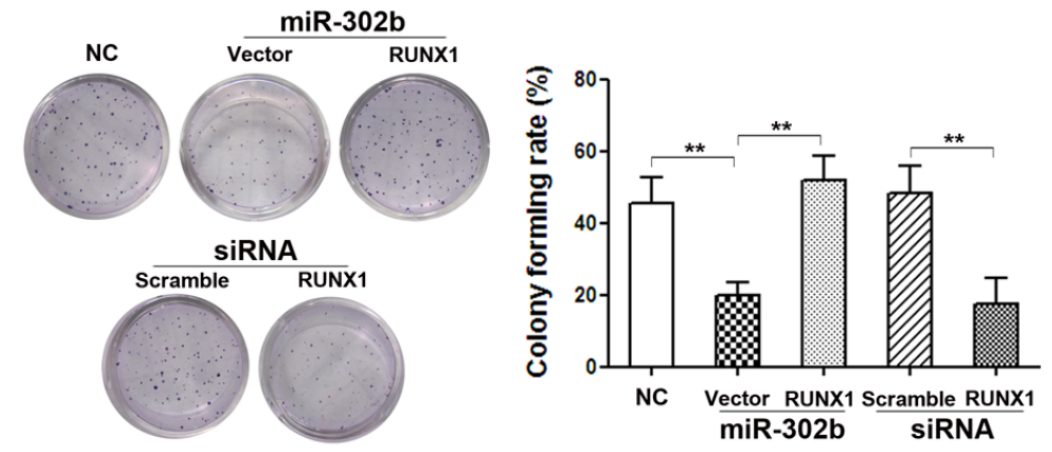

$\mathbf{E}$
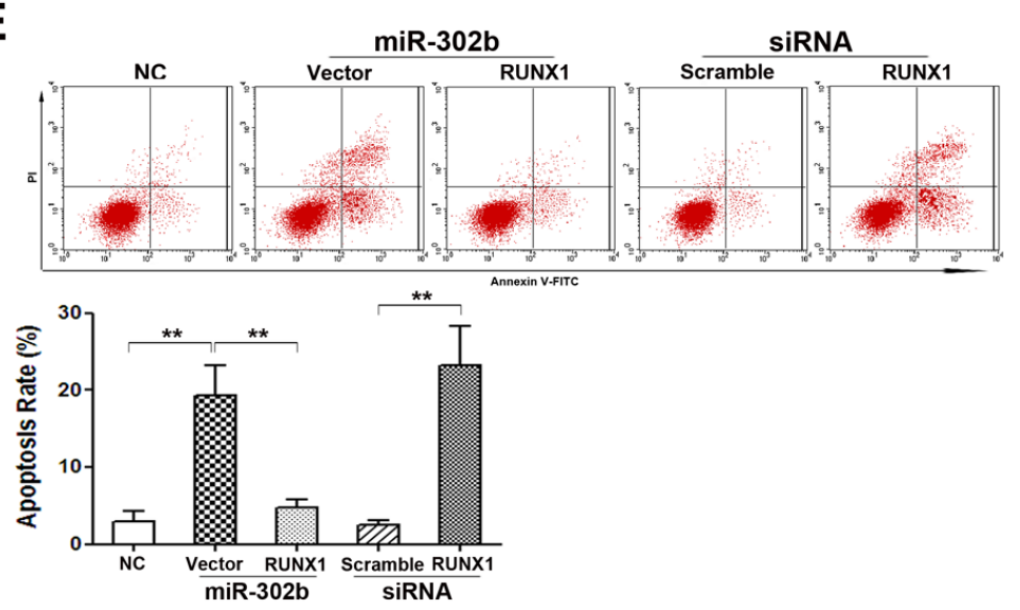

Fig. 4. RUNX1 mediates miR-302b induced growth inhibition and apoptosis promotion. A. The protein levels of RUNX1 were measured in SKOV3 cells co-transfected with miR-302b and a RUNX1 mutant construct lacking the 3'UTR, and in RUNX1 siRNA transfected cells. B. Cell viability was measured by MTT assay. C. Colony formation assay. D. Cell cycle progression. E. Cell apoptosis by Annexin V/FITC flow cytometry. ${ }^{* *} P<0.01$.

and its target RUNX1 on the activity of STAT3 and its downstream effectors in SKOV3 cells. Western blot analysis showed that miR-302b downregulated phospho-STAT3, Cyclin D1 and Bcl-2 and upregulated the pro-apoptotic protein Bax and Cleaved caspase 3, whereas anti- 
Fig. 5. Overexpression of miR302b inhibits the STAT3 pathway. A. SKOV3 cells were transfected with miR-302b, anti-miR-302b, RUNX1 siRNA, or their respective nonspecific controls, and the expression of phospho STAT3 ( $p$ STAT3), Cyclin D1, Bcl-2, Bax, and Cleaved caspase 3 were analyzed by western blotting. Representative blots and the results of densitometric analyses are shown. ${ }^{*} P<0.05,{ }^{* *} P<0.01,{ }^{* * *} P<0.001$.

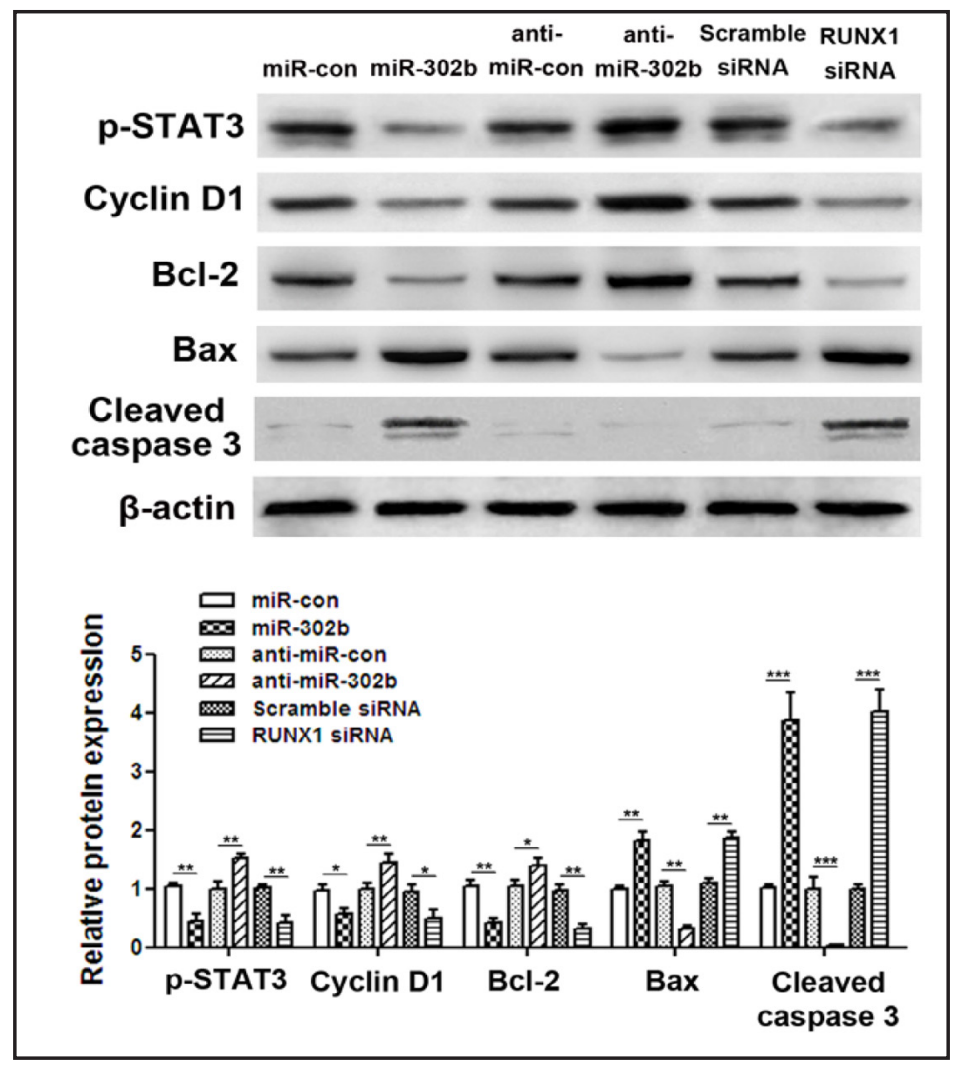

Fig. 6. MiR302b suppresses SKOV3 xenograft tumor growth in nude mice. A. Tumor volumes over 32 days of observation. B. All animals were sacrificed on day 32 and tumors were removed and weighted. Data represent mean \pm S.D. from at least 5 mice per group. C. Representative Ki67- and PCNAstained sections of xenograft tu-

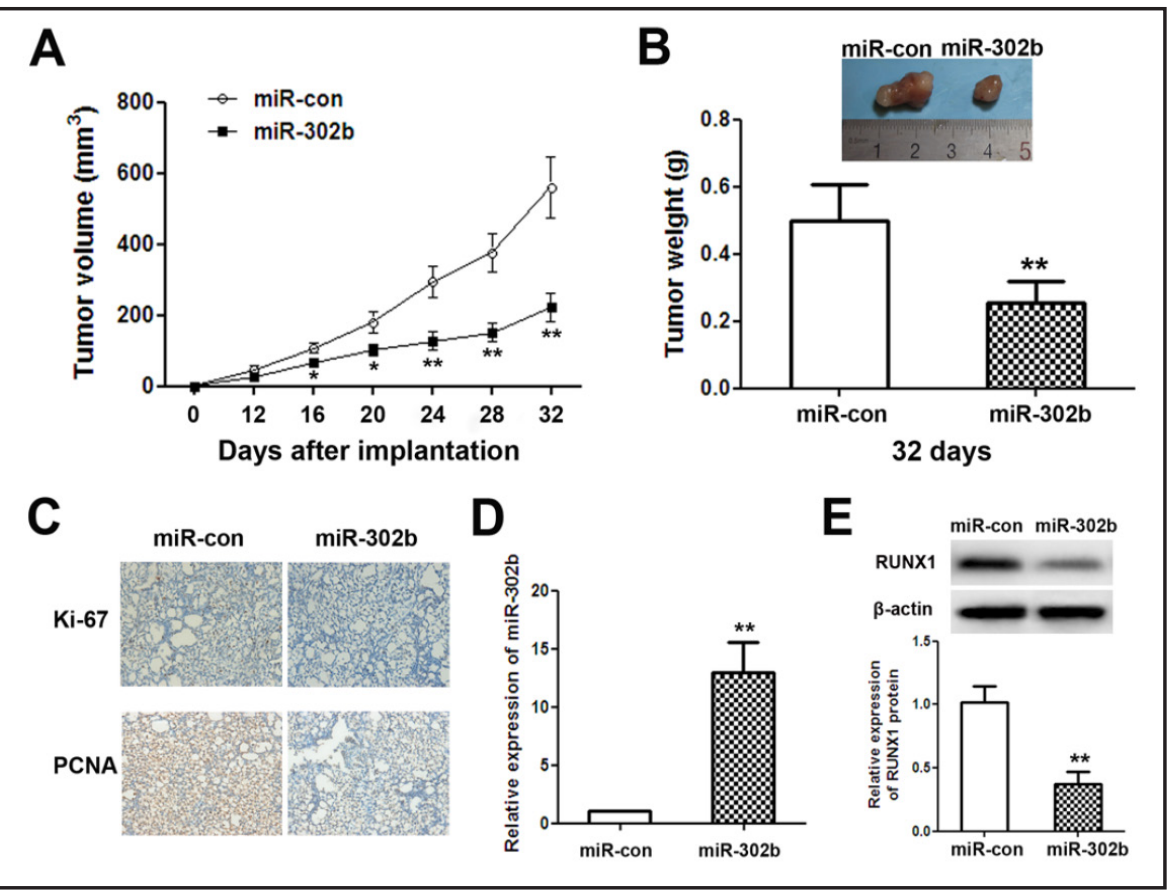

mors. D. MiR-302b mRNA levels in xenograft tumor tissues as determined by qRT-PCR. E. RUNX1 protein levels in miR-302b-overexpressing and control xenograft tumor tissues determined by western blotting. ${ }^{*} P<0.05,{ }^{* *} P<0.01$.

miR-302b had the opposite effects (Fig. 5). Knockdown of RUNX1 suppressed the activation of STAT3 and its downstream effectors, downregulated Cyclin D1 and promoted apoptosis, similar to the effect of miR-302b overexpression. Taken together, these results suggested 
that miR-302b acts as a tumor suppressor in ovarian cancer by modulating the activity of the STAT3 pathway through its target RUNX1.

\section{MiR-302b suppresses tumor growth in vivo}

To examine the effect of miR-302b in vivo, a tumor xenograft model was established using miR-302b overexpressing cells and tumor growth was monitored over a period of 32 days. Tumor growth was significantly inhibited in mice inoculated with miR-302b overexpressing cells compared to vector control inoculated mice (Fig. 6A). Tumor weight at 32 days was approximately 50\% lower in mice inoculated with miR-302b-expressing cells than in those inoculated with control vector-transfected cells $(P<0.01)$ (Fig. 6B). Immunohistochemical analysis showed lower levels of Ki-67 antigen-positive cells and PCNA staining in tissues of mice inoculated with miR-302b expressing cells than in vector control expressing tumors, indicating a lower rate of cell proliferation in miR-302b overexpressing tumors (Fig. 6C). Analysis of miR-302b expression in tumor tissues by qRT-PCR showed significantly higher levels of miR-302b mRNA in tumors generated from miR-302b overexpressing cells (Fig. 6D). Conversely, RUNX1 expression was significantly lower in miR-302b overexpressing tumors than in the controls, as determined by western blotting (Fig. 6E).

\section{Discussion}

In the present study, we investigated the role of miR-302b in EOC, which led to the identification of RUNX1 as its direct target and the elucidation of a potential mechanism underlying the tumor suppressor function of miR-302b. Over 60 miRNAs have been predicted to target RUNX1, and several of these have been validated including miRNAs 17, 20a, 18a, 27a, 30c and 106a [20]. The present study is the first to show the modulation of RUNX1 expression by miR-302b in EOC. We showed that miR-302b is downregulated in EOC tissues in association with RUNX1 upregulation, and ectopic expression of miR-302b inhibited cell proliferation and colony formation and induced cell cycle arrest and apoptosis via the downregulation of its target RUNX1. Although RUNX1 can act as a tumor suppressor or an oncogene, its critical role in cancer cell growth and survival has been demonstrated in several solid tumors [5]. In skin squamous cell carcinoma, RUNX1 is overexpressed and its action is thought to be mediated by the repression of $\mathrm{p} 21$ and activation of STAT3. RUNX1 is associated with tumorigenesis in colon cancer, whereas in breast cancer, its role as a tumor suppressor or oncogene remains unclear $[21,22]$. RUNX1 is highly overexpressed in invasive endometrial carcinoma, and the colocalization, upregulation and codistribution of RUNX1 with matrix metalloproteases in both endometrioid endometrial and ovarian endometrioid carcinoma (OEC) suggests that it plays a role in the progression and invasion of OEC [23, 24]. In an analysis of primary cultures derived from patients with EOC, RUNX1 overexpression was associated with advanced (metastatic) EOC [25], and the oncogenic role of RUNX1 in EOC was confirmed in SKOV3 cells, in which RUNX1 promoted cell proliferation, cell cycle progression and migration/invasion [6]. RUNX1 is overexpressed in various epithelial tumors including EOC and its association with tumor initiation was shown to be mediated by a RUNX1/STAT3 signaling axis [6].

Constitutive activation of STAT3, which has been detected in several cancers including ovarian cancer, can lead to cellular transformation and tumorigenesis [26]. Furthermore, STAT3 activation is correlated with disease stage, lymph node metastasis, drug resistance and poor survival in ovarian cancer [27-29]. The expression of the phosphorylated form of STAT3, p-STAT3, is higher in ovarian cancer cell lines and in EOC tissues compared to benign ovarian tumors or normal ovarian tissues $[27,30]$. The nuclear localization of p-STAT3 has been detected in a significant proportion of ovarian cancer patients in association with poor prognosis $[28,31]$. Treatment with the STAT3 inhibitor corosolic acid inhibited EOC cell proliferation and increased the sensitivity of EOC cells to anticancer drugs in a recent study, confirming the link between STAT3 activity and tumorigenesis in EOC [32]. In the present 
study, we showed that overexpression of miR-302b decreased the levels of p-STAT3 as well as those of its downstream effectors in SKOV3 cells, indicating that the tumor suppressor effects of miR-302b may be mediated by the inactivation of STAT3 in EOC.

In conclusion, in the present study we showed that miR-302b is downregulated in EOC and its ectopic expression inhibits cell proliferation and colony formation and induces cell cycle arrest and apoptosis in ovarian cancer cells. We identified RUNX1 as a direct target of miR-302b, and showed that silencing of RUNX1 mimicked the effects of miR$302 \mathrm{~b}$ overexpression in EOC, indicating that the tumor suppressor effects of miR-302b are mediated by the downregulation of RUNX1. MiR-302b inhibited the STAT3 signaling pathway, and RUNX1 silencing had a similar effect, demonstrating that miR-302b regulates STAT3 signaling through the modulation of its target gene RUNX1. Our results provide insight into the role of miRNAs in EOC tumorigenesis and reveal a novel mechanism involving the transcription factor RUNX1 and STAT3, suggesting potential prognostic biomarkers and therapeutic targets for the treatment of ovarian cancer.

\section{Acknowledgments}

This work was supported by a grant from Heilongjiang Special Funds for Outstanding Youth (No. JC201108), Heilongjiang Province, China.

\section{References}

1 Ito Y: RUNX genes in development and cancer: regulation of viral gene expression and the discovery of RUNX family genes. Adv Cancer Res 2008;99:33-76.

2 Wang CQ, Jacob B, Nah GS, Osato M: Runx family genes, niche and stem cell quiescence. Blood Cells Mol Dis 2010;44:275-286.

- 3 Bernardin-Fried F, Kummalue T, Leijen S, Collector MI, Ravid K, Friedman AD: AML1/RUNX1 increases during G1 to S cell cycle progression independent of cytokine-dependent phosphorylation and induces cyclin D3 gene expression. J Biol Chem 2004;279:15678-15687.

4 Strom DK, Nip J, Westendorf JJ, Linggi B, Lutterbach B, Downing JR, Lenny N, Hiebert SW: Expression of the AML-1 oncogene shortens the G(1) phase of the cell cycle. J Biol Chem 2000;275:3438-3445.

5 Scheitz CJ, Tumbar T: New insights into the role of Runx1 in epithelial stem cell biology and pathology. J Cell Biochem 2013;114:985-993.

6 Scheitz CJ, Lee TS, McDermitt DJ, Tumbar T: Defining a tissue stem cell-driven Runx1/Stat3 signalling axis in epithelial cancer. EMBO J 2012;31:4124-4139.

7 Bromberg JF, Wrzeszczynska MH, Devgan G, Zhao Y, Pestell RG, Albanese C, Darnell JE Jr: Stat3 as an oncogene. Cell 1999;98:295-303.

-8 Bowman T, Garcia R, Turkson J, Jove R: STATs in oncogenesis. Oncogene 2000;19:2474-2488.

9 Zhao WH, Hao M: Progress in research on Stat3 in gynecological malignant tumors. Chin J Woman Child Health Res 2008;18:440-443.

10 Yu H, Pardoll D, Jove R: STATs in cancer inflammation and immunity: a leading role for STAT3. Nat Rev Cancer 2009;9:798-809.

-11 Keita M, Bachvarova M, Morin C, Plante M, Gregoire J, Renaud MC, Sebastianelli A, Trinh XB, Bachvarov D: The RUNX1 transcription factor is expressed in serous epithelial ovarian carcinoma and contributes to cell proliferation, migration and invasion. Cell Cycle 2013;12:972-986.

12 Wang D, Qiu C, Zhang H, Want J, Cui Q Yin Y: Human microRNA oncogenes and tumor suppressors show significantly different biological patterns" from functions to targets. PloS One 2010;5(9) pii:e13067.

13 Parikh A, Lee C, Peronne J, Marchini S, Baccarini A, Kolev V, Romualdi C, Fruscio R, Shah H, Wang F, Mullokandov G, Fishman D, D'Incalci M,Rahaman J, Kalir T, Redline RW, Brown BD, Narla G, DiFeo A: microRNA-181a has a critical role in ovarian cancer progression through the regulation of the epithelialmesenchymal transition. Nat Commun 2014;5:2977. 


\section{Cellular Physiology Cell Physiol Biochem 2014;34:2209-2220 and Biochemistry

14 Iorio MV, Visone R, Di Leva G, Donati V, Petrocca F, Casalini P, Taccioli C, Volinia S, Liu CG, Alder H, Calin GA, Ménard S, Croce CM: MicroRNA signatures in human ovarian cancer. Cancer Res 2007;67:8699-8707.

15 Nam EJ, Yoon H, Kim SW, Kim H, Kim YT, Kim JH, Kim JW, Kim S: MicroRNA expression profiles in serous ovarian carcinoma. Clin Cancer Res 2008;14:2690-2695.

16 Dahiya N, Morin PJ: MicroRNAs in ovarian carcinomas. Endocr Relat Cancer 2010;17:F77-F89.

17 Yan GJ, Yu F, Wang B, Zhou HJ, Ge QY, Su J, Hu YL, Sun HX, Ding LJ: MicroRNA miR-302 inhibits the tumorigenicity of endometrial cancer cells by suppression of Cyclin D1 and CDK1. Cancer Lett 2014;345:39-47.

18 Zhang M, Yang Q, Zhang L, Zhou S, Ye W, Yao Q, Li Z, Huang C, Wen Q, Wang J: miR-302b is a potential molecular marker of esophageal squamous cell carcinoma and functions as a tumor suppressor by targeting ErbB4. J Exp Clin Cancer Res 2014;33:10.

19 Wang L, Yao J, Shi X, Hu L, Li Z, Song T, Huang C: MicroRNA-302b suppresses cell proliferation by targeting EGFR in human hepatocellular carcinoma SMMC-7721 cells. BMC Cancer 2013;13:448.

20 Zhang L, Huang J, Yang N, Greshock J, Megraw MS, Giannakakis A, Liang S, Naylor TL, Barchetti A, Ward MR, Yao G, Medina A, O'brien-Jenkins A, Katsaros D, Hatzigeorgiou A, Gimotty PA, Weber BL, Coukos G: microRNAs exhibit high frequency genomic alterations in human cancer. Proc Natl Acad Sci U S A 2006;103:9136-9341.

21 Rossetti S, Sacchi N: RUNX1: A microRNA hub in normal and malignant hematopoiesis. Int J Mol Sci 2013;14:1566-88.

22 Pradhan MP, Prasad NK, Palakal MJ. A systems biology approach to the global analysis of transcription factors in colorectal cancer. BMC Cancer 2012;12:331.

23 Wang L, Brugge JS, Janes KA: Intersection of FOXO- and Runx1-mediated gene expression programs in single breast epithelial cells during morphogenesis and tumor progression. Proc Natl Acad Sci 2011;108:E803-E812.

24 Planagumà J, Diaz-Fuertes M, Gil-Moreno A, Abal M, Monge M, García A, Baró T, Thomson TM, Xercavins J, Alameda F, Reventós J: A differential gene expression profile reveals overexpression of RUNX1/AML1 in invasive endometrioid carcinoma. Cancer Res 2004;64:8846-8853.

25 Planagumà J, Liljeström M, Alameda F, Bützow R, Virtanen I, Reventós J, Hukkanen M: Matrix metalloproteinase-2 and matrix metalloproteinase-9 codistribute with transcription factors RUNX1/AML1 and ETV5/ERM at the invasive front of endometrial and ovarian carcinoma. Hum Pathol 2011;42:57-67.

-26 Keita M, Wang ZQ, Pelletier JF, Bachvarova M, Plante M, Gregoire J, Renaud MC, Mes-Masson AM, Paquet ÉR, Bachvarov D: Global methylation profiling in serous ovarian cancer is indicative for distinct aberrant DNA methylation signatures associated with tumor aggressiveness and disease progression. Gynecol Oncol 2013;128:356-363.

27 Rath KS, Naidu SK, Lata P, Bid HK, Rivera BK, McCann GA, Tierney BJ, Elnaggar AC, Bravo V, Leone G, Houghton P, Hideg K, Kuppusamy P, Cohn DE, Selvendiran K: HO-3867, a safe STAT3 inhibitor, is selectively cytotoxic to ovarian cancer. Cancer Res 2014;74:2316-2327.

28 Min H, Wei-hong Z: Constitutive activation of signal transducer and activator of transcription 3 in epithelial ovarian carcinoma. J Obstet Gynaecol Res 2009;35:918-925.

-29 Rosen DG, Mercado-Uribe I, Yang G, Bast RC Jr, Amin HM, Lai R, Liu J: The role of constitutively active signal transducer and activator of transcription 3 in ovarian tumorigenesis and prognosis. Cancer 2006;107:2730-2740.

-30 Duan Z, Foster R, Bell DA, Mahoney J, Wolak K, Vaidya A, Hampel C, Lee H, Seiden MV: Signal transducers and activators of transcription 3 pathway activation in drug-resistant ovarian cancer. Clin Cancer Res 2006;12:5055-5063.

31 Huang M, Page C, Revnolds RK, Lin J: Constitutive activation of stat3 oncogene product in human ovarian carcinoma cells. Gynecol Oncol 2000;79:67-73.

-32 Colomiere M, Ward AC, Riley C, Trenerry MK, Cameron-Smith D, Findlay J, Ackland L, Ahmed N: Cross talk of signals between EGFR and IL-6R through JAK2/STAT3 mediate epithelial-mesenchymal transition in ovarian carcinomas. Br J Cancer 2009;14:134-144.

-33 Fujiwara Y, Takaishi K, Nakao J, Ikeda T, Katabuchi H, Takeya M, Komohara Y: Corosolic acid enhances the antitumor effects of chemotherapy on epithelial ovarian cancer by inhibiting signal transducer and activator of transcription 3 signaling. Oncol Lett 2013;6:1619-1623. 